\title{
Three-dimensional Visible Light Positioning: an Experimental Assessment of the Importance of the LEDs' Locations
}

\author{
D. Plets*, S. Bastiaens*, M. Ijaz ${ }^{\dagger}$, Y. Almadani ${ }^{\dagger}$, L. Martens*, W. Raes ${ }^{\ddagger}$, N. Stevens ${ }^{\ddagger}$, W. Joseph*, \\ * Information Technology Department, imec-Ghent University \\ Technologiepark 126, B-9052 Ghent, Belgium \\ $\dagger$ School of Engineering, Engineering and Materials Research Centre, Manchester Metropolitan University \\ John Dalton Building, Chester Street, M1 5GD, Manchester, UK \\ $\ddagger$ KULeuven-ESAT-DraMCo \\ Gebroeders de Smetstraat 1, B-9000 Ghent, Belgium \\ david.plets@ugent.be
}

\begin{abstract}
This paper assesses the accuracy of a threedimensional Visible Light Positioning (VLP) algorithm for two different Light Emitting Diode (LED) configurations using the same four LEDs, but mounted at different locations on the ceiling. The two configurations are both simulated and measured at 22801 test points. It is observed that a classic square LED configuration results in position ambiguities, causing errors up to several meters. Alternatively, a star-shaped LED configuration is able to uniquely reconstruct the photodiode's location. For LEDs at a height of approximately $3 \mathrm{~m}$ above the receiver, median errors of $12.7 \mathrm{~cm}$ and maximal errors of $21.1 \mathrm{~cm}$ are experimentally obtained, showcasing the applicability of 3D VLP for drone navigation.
\end{abstract}

\section{INTRODUCTION}

Indoor localization research has gained a lot of attention during the last decade, with possible applications in various markets. Depending on the use case, different accuracies are desired. E.g, for navigating humans through buildings, errors up to a few metres can be tolerated [1]. On the other hand, Automated Guided Vehicles in industrial environments should be able to position themselves within only a few centimetres or even millimetres. As such, research is still vibrant around many different positioning techniques: Received Signal Strength (RSS)-based Radiofrequent (RF)-based positioning [1], camera-based positioning [2], laser-based positioning, etc. A popular research topic is RF-based Time-of-Flightbased positioning using Ultra-Wideband (UWB) signals, yielding accuracies below $10 \mathrm{~cm}$ [3]. A possible competitor for UWB-positioning is RSS-based Visible Light Positioning (VLP), able to deliver a similar performance. In [4], UWB has been investigated to localize or navigate an Unmanned Aerial Vehicle (UAV) or drone. In [5], a 3D VLP positioning approach based on Artificial Neural Networks is proposed. Yang et al. [6] proposed an RSS-based 3D VLP positioning algorithm. In [7], an efficient VLP algorithm was presented to obtain a $3 \mathrm{D}$ position, also making it suitable for drone navigation. A four-LED configuration was investigated, with the receiver module not requiring additional height sensors. However, simulations indicated that a classic configuration with four Light Emitting Diodes (LEDs) mounted in a squareshape is not able to unambiguously solve the $3 \mathrm{D}$ position. As a better alternative, a star-shaped configuration was proposed. As the square LED configuration is assumed in many research papers [8], [9], it is important to also compare the squareshaped and star-shaped configuration in an experimental way. This paper will therefore assess realistic accuracies for 3D VLP for two LED configurations. Quite some research is available on network planning and the optimal placement of base stations for RF communication [10], [11], or for optimal node placement for RF-based localization [12], [13]. However, to the authors' knowledge, no research has yet been performed on optimal LED placement for a maximal VLP accuracy.

The outline of this paper is as follows. Section II presents the VLP configuration that is both simulated and experimentally assessed, while Section III describes the assumed channel model for the simulations. In Section IV, the employed localization algorithms are described. In Section V, the results are discussed and the main findings of this paper are summarized in Section VI.

\section{VLP CONFIGURATION}

The $3 \mathrm{D}$ positioning performance is evaluated within a VLP lab, measuring $4 \mathrm{~m} \times 4 \mathrm{~m}$. Fig. 1 shows a picture of the lab. The LEDs' height can be adjusted, up to a height of $5 \mathrm{~m}$. Reflections from walls or objects are avoided by using black cloth as walls of the lab area. Four BXRE-50C3001-D-24 LEDs are intensity-modulated, transmitting pulse trains with a duty cycle of 0.5 . Their current magnitudes are $[600,750,750$, 750] $\mathrm{mA}$ and their frequencies are chosen as $f_{i}=2^{i-1} \cdot f_{0}$, with $i=1 . .4$ and $f_{0}$ equal to $500 \mathrm{~Hz}$. As such, the contributions of the different LEDs can be demultiplexed at the receiver side [14].

The photodiode (PD) receiver is a Thorlabs PDA36A2 commercial photodiode with an active area $A_{R}$ equal to 


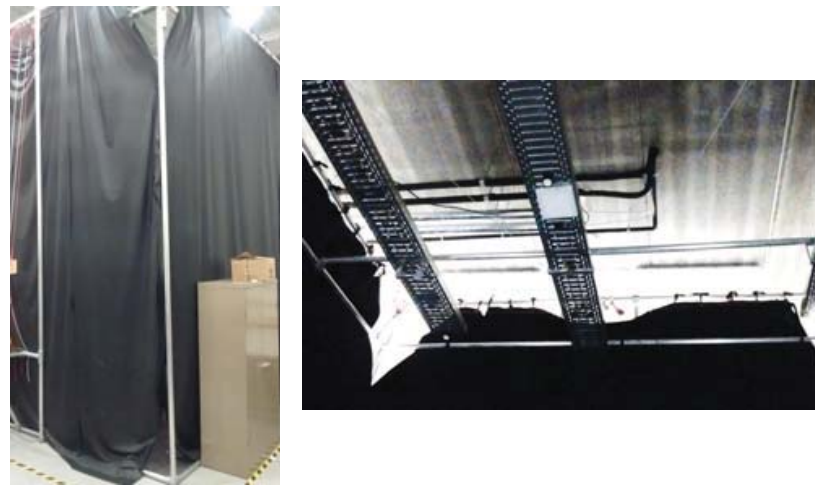

Fig. 1: Exterior view of the VLP lab with the black cloth sides, and interior view of ceiling rails for attaching LEDs.

$13 \mathrm{~mm}^{2}$. The PD is attached to a one-by-one meter twodimensional slider system (Velmex BiSlides) with a measurement granularity of $2.5 \mathrm{~cm}$, and an accuracy better than $1 \mathrm{~mm}$. By subsequently displacing the entire slider system in the receiver plane, the entire four-by-four meter plane is measured, resulting in $151^{2}$ measurement locations. The photocurrents are digitized using National Instrument's USB6212 DAQ Device. MATLAB is used to for the FFT-based demodulation into received power values per LED, as specified in [14]. To reduce the impact of noise, fifteen power values are averaged per measurement location. Given that the lowest used frequency is $500 \mathrm{~Hz}$, a new location is obtained each $30 \mathrm{~ms}$. The height of the PD is $0.21 \mathrm{~m}$ during all tests. Despite the receiver height being fixed, the positioning algorithm will assume the PD height to be unknown.

As already introduced, two four-LED configurations will be assessed and compared. A top view of the LEDs' locations in the considered environment is shown in Fig. 2, for both configuration 1 ('Square', blue dots) and configuration 2 ('Star', red dots). Table I lists shows the (x,y,z) coordinates of the four LEDs in both configurations. These coordinates are determined using a laser-meter.

- 'Square' - In the first configuration, denoted as 'Square', the LEDs are mounted at the four corners of a square, a typical configuration assumed in many research papers. It can also be seen as a configuration with four LEDs at the same distance of the square centre ('concyclic' locations), but azimuthally separated by $90^{\circ}$.

- 'Star' - The second configuration, denoted as 'Star', will have its four LEDs mounted in a star-shaped fashion, with one central LED, surrounded by the three other LED at more or less the same distance from the centre LED, but azimuthally separated by $120^{\circ}$.

\section{ChANNEL MODEL}

This section will clarify the channel model that will be assumed for the simulations. We only consider the Line-ofSight (LoS) path between a LED and the PD. Research on the impact of (and possible ways of compensating for) reflections

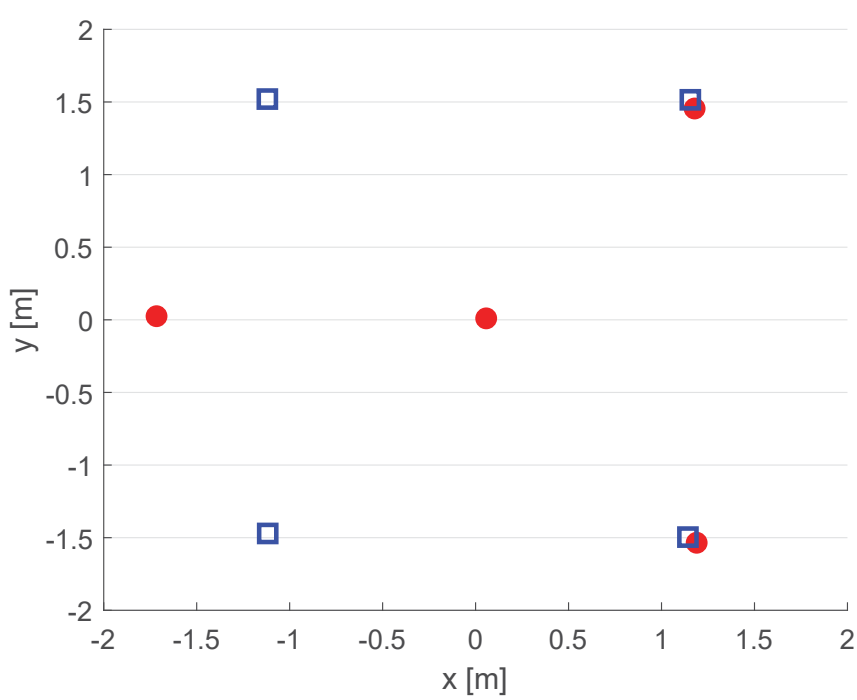

Fig. 2: Top view of LEDs' locations in the considered environment, for configuration 'Square' (blue squares) and 'Star' (red dots).

TABLE I: (x,y,z) coordinates of the 4 LEDs for configuration 'Square' and for configuration 'Star'

\begin{tabular}{|l|r|l|r|r|r|r|r|l|}
\hline \multirow{2}{*}{} & \multicolumn{4}{|c|}{ Square } & \multicolumn{4}{c|}{ Star } \\
\cline { 2 - 10 } & L1 & L2 & L3 & L4 & L1 & L2 & L3 & L4 \\
\hline $\mathrm{x}(\mathrm{m})$ & -1.12 & 1.16 & -1.12 & 1.14 & 1.18 & -1.72 & 1.19 & 0.06 \\
\hline $\mathrm{y}(\mathrm{m})$ & -1.47 & 1.52 & 1.52 & -1.50 & 1.46 & 0.03 & -1.54 & 0.01 \\
\hline $\mathrm{z}(\mathrm{m})$ & 3.22 & 3.23 & 3.20 & 3.21 & 3.25 & 3.19 & 3.20 & 3.18 \\
\hline
\end{tabular}

will be considered as future work. Fig. 3 defines the model parameters of the visible light channel. The power $P_{R}$ received at the PD is calculated according to the following channel model:

$$
P_{R}=P_{E} \cdot h_{L o S}+P_{N},
$$

with $P_{E}$ the emitted optical power by the LED and $P_{N}$ the noise power. $h_{L o S}$ is the channel gain along the direct link and can be described as follows, when assuming a Lambertian radiator:

$$
h_{L o S}=\frac{m+1}{2 \pi d^{2}} \cos ^{m}(\phi) \cdot A_{R} \cdot \cos (\psi) \cdot T_{R}(\psi) \cdot G_{R}(\psi),
$$

with $m$ the order of the Lambertian emitter, and $\phi$ the angle of irradiance (i.e., the angle between the LED normal and the vector $\vec{v}_{L E D 2 P D}$ from the LED to the PD. $\cos (\psi)$ represents the cosine model for the receiver's angular responsivity. $T_{R}(\psi)$ and $G_{R}(\psi)$ are the optical filter's gain and the optical concentrator's gain at the receiver, respectively, with $\psi$ the angle of incidence (i.e., the angle between the PD normal $\vec{n}_{P D}$ and the vector from the PD to the LED). The LEDs will be assumed to be within the field-of-view (FOV) of the PD and $T_{R}(\psi)$ and $G_{R}(\psi)$ are assumed equal to $1 . d$ is the distance between the LED and the PD, and $A_{R}$ the actual PD area.

LED and PD will be assumed to be horizontally oriented. 


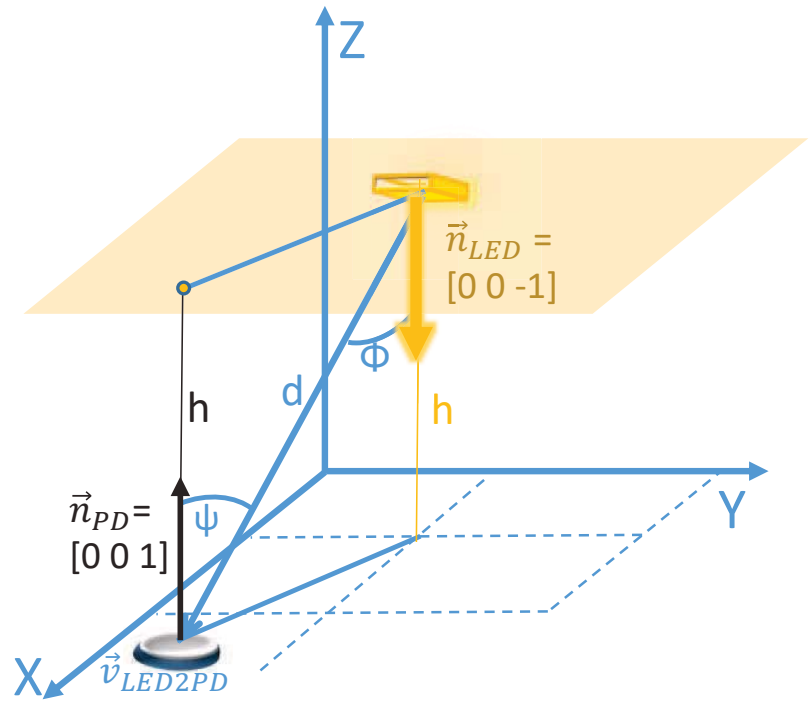

Fig. 3: Overview of visible light channel.

For an untilted LED (i.e., horizontally oriented), the angle of irradiance $\phi=\psi$ (see Fig. 3) with $\cos (\phi)=\cos (\psi)$ is equal to $h / d$, with $h$ the height difference between the LED and the PD. Note that the assumption of the PD being located in the xy-plane (see Fig. 3), does not retract from the generality of the work, as only the LED-PD height difference $h$ matters. For the conducted simulations, $\mathrm{m}$ will be assumed equal to 1 , and $P_{E}$ of each of the LEDs will be estimated as $P_{E}^{e s t}$, by conducting one measurement right below the LED, such that $P_{E}^{e s t}=\frac{P_{R}}{A_{R}} \frac{m+1}{2 \pi d^{2}}$. As such, the four LED powers $P_{E}$ were estimated at 13.6, 17.0, 16.7, and 16.2 W for LEDs L1, L2, L3, L4 respectively.

\section{A. Noise model for simulations}

In [9], a noise model is proposed, where the variance is proportional to the received power $P_{R}$ :

$\sigma_{\text {total }}^{2}=\frac{8 \pi k T \eta A_{R} I_{2} B^{2}}{G}+\frac{16 \pi^{2} k T \gamma_{L} \eta^{2} A_{R}^{2} I_{3} B^{3}}{g_{m}}+2 q \gamma P_{R} B$

where we assume that only VLP-modulated light is present (background current is zero). This is a fair assumption in industrial warehouse environments and is also valid in our experimental configuration (see Section II). Detailing each of the parameters in eq. (3) would lead us too far, the reader is refered to [9] for more info. However, as many of these parameters are unknown in our system, we experimentally estimate a suitable noise model for our system simulations. At all evaluation locations, 15 photocurrent samples are collected, from which each time a standard deviation value of the noise is estimated. Fig. 4 shows the estimated noise variance $\sigma^{2}$ as a function of the photocurrent $I_{P D}=\gamma \cdot P_{R}$, with $\gamma$ the PD's responsivity. The figure shows no clear increase with photocurrent, meaning that here, the noise is dominated by other factors than the photocurrent. Hence, for our system,

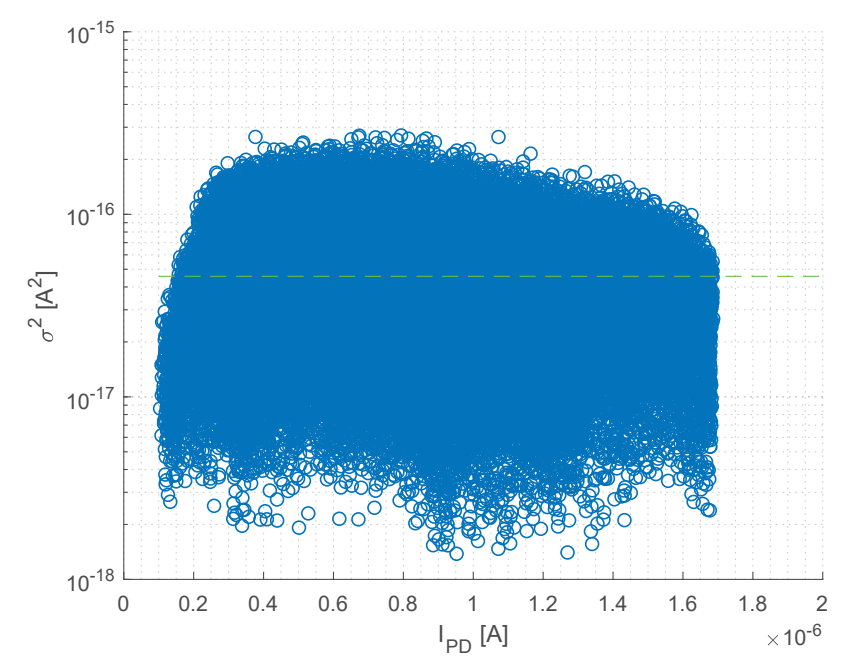

Fig. 4: Experimentally obtained noise variance at all locations for the considered VLP configuration.

we will assume the noise variance to be equal to the average value of all estimations, i.e., $\sigma^{2}=4.57 \cdot 10^{-17} \mathrm{~A}^{2}$. Linking to eq. (1), $\sigma_{P_{N}}^{2}$ is calculated as $\sigma^{2} / \gamma^{2}$, with $\gamma$ estimated at 0.22 here, by weighing the PD's responsivity spectrum with the LED spectrum.

\section{Positioning Algorithms}

We apply a trilateration algorithm to estimate the position based on the observed light intensities, both from simulations and experiments. We distinguish between a 2D trilateration algorithm and a 3D trilateration algorithm. While the 2D algorithm assumes the PD height to be exactly known, the 3D algorithm assumes no prior knowledge of the PD height, and thus has an additional degree of freedom. In [7], a 3D trilateration algorithm was presented. The algorithm encompasses the calculation of 2D trilateration estimates for a range of possible PD heights, after which these estimates are evaluated based on a Non-Linear Least Squares (NLLS) minimization. When limiting this range of possible PD heights to one value - the known height - the 3D algorithm thus reduces to the $2 \mathrm{D}$ trilateration algorithm.

The inputs to the 2D trilateration for an assumed LED-PD height difference $h_{i}$ are estimated distances between a $\mathrm{LED}_{\mathrm{i}}$ and the unknown PD location, based on the observed received power $P_{R i}$ from $\mathrm{LED}_{\mathrm{i}}$. These distances $\widehat{d}_{i}$ are estimated by solving eq. (2) for $d_{i}$ :

$$
\widehat{d}_{i}=\sqrt[m+3]{\frac{(m+1) A_{R} P_{E i} h_{i}{ }^{m+1}}{2 \pi P_{R i}}}
$$

with $P_{E i}$ the power emitted by $\mathrm{LED}_{\mathrm{i}}$, and $h_{i}$ the known height difference between $\mathrm{LED}_{\mathrm{i}}$ and PD (see also Fig. 3). It is observed that the trilateration process delivers the best positioning performance when all four sources are used in the 2D trilateration algorithm, so in the analysis of this paper, we will also use all four LED sources for the $2 \mathrm{D}$ trilateration. 
The 3D trilateration builds on an iterative evaluation of $2 \mathrm{D}$ trilateration outcomes for different assumed LED-PD height differences $h$ [7]. For each $h$ value, the following cost function $C(h)$ is evaluated:

$$
\begin{aligned}
C(h)= & \frac{1}{N} \sum_{i=1}^{N}\left[\widehat{d}_{i}(h)\right. \\
& \left.-\sqrt{\left(\widehat{x}(h)-x_{i}\right)^{2}+\left(\widehat{y}(h)-y_{i}\right)^{2}+(h)^{2}}\right]^{2}
\end{aligned}
$$

with $N$ the total number of LEDs ( $N=4$ here), $\widehat{d}_{i}(h)$ is the distance that is estimated for the observed received power $P_{R i}$ (see eq. (4)) for the assumed LED-PD height difference $h$. $(\widehat{x}(h), \widehat{y}(h))$ is the output of the $2 \mathrm{D}$ trilateration algorithm that is used, assuming the PD height to be $h_{P D}=h_{L E D}-h$. As such, $C(h)$ calculates, for each $h$, the average squared error between the estimated LED-PD distances $\widehat{\vec{d}}_{i}(h)$ ('estimated distance of LED to PD location') and the distances between the LEDs and the 2D-estimated PD location ('distance of LED to estimated PD location'). The $h$ value for which $C(h)$ is minimal, yields the estimated LED-PD height difference, and the $2 \mathrm{D}$ trilateration for this height difference then delivers the estimated $(\widehat{x}(h), \widehat{y}(h))$ location. In this work, we will use the trilateration algorithm described in [7] (using four LED sources), but it should be noted that the NLLS minimization described above can be used in conjunction with any $2 \mathrm{D}$ trilateration algorithm.

TABLE II: Simulated and experimentally obtained median $\left(\mathrm{p}_{50}\right)$ and maximal $\left(\mathrm{p}_{95}\right)$ positioning errors, for the two LED configurations (square- and star-shaped), and for 3D (PD height unknown) and 2D (PD height exactly known) localization.

\begin{tabular}{|l|c|l|l|l|}
\hline \multirow{2}{*}{ p50 error $(\mathbf{c m})^{\mid}$} & \multicolumn{2}{|c|}{ Square } & \multicolumn{2}{c|}{ Star } \\
\cline { 2 - 5 } & 2D & 3D & 2D & 3D \\
\hline Experimental & 13.4 & 222.6 & 15.3 & 12.7 \\
\hline Simulated & 2.5 & 212.3 & 6.7 & 3.2 \\
\hline \hline \multirow{2}{*}{ p95 error (cm) } & \multicolumn{2}{|c|}{ Square } & \multicolumn{2}{c|}{ Star } \\
\cline { 2 - 5 } & 2D & 3D & 2D & 3D \\
\hline Experimental & 28.7 & 277.8 & 25.7 & 21.1 \\
\hline Simulated & 7.4 & 276.2 & 8.9 & 7.7 \\
\hline
\end{tabular}

\section{RESUlts}

Table II lists the median ( $\mathrm{p}_{50}$ ) and maximal ( $\left.\mathrm{p}_{95}\right)$ positioning errors for the different algorithms ('2D' and '3D') and for the two LED configurations ('star' and 'square'), obtained via simulations or via experiments. Fig. 5 shows the simulated ('sim') and experimental ('exp') cumulative distribution function (cdf) of the positioning error using the square-shaped LED configuration, both for the 3D estimation and for the 2D estimation (assuming the height to be exactly known). Fig. 5 and Table II show the small positioning errors when the height is known ('2D sim' and '2D exp'): a median of 2.5 and $13.4 \mathrm{~cm}$ for simulations and experiments respectively. As found via simulations in [7], 3D location estimations indeed suffer from a possible ambiguity when the four LEDs are

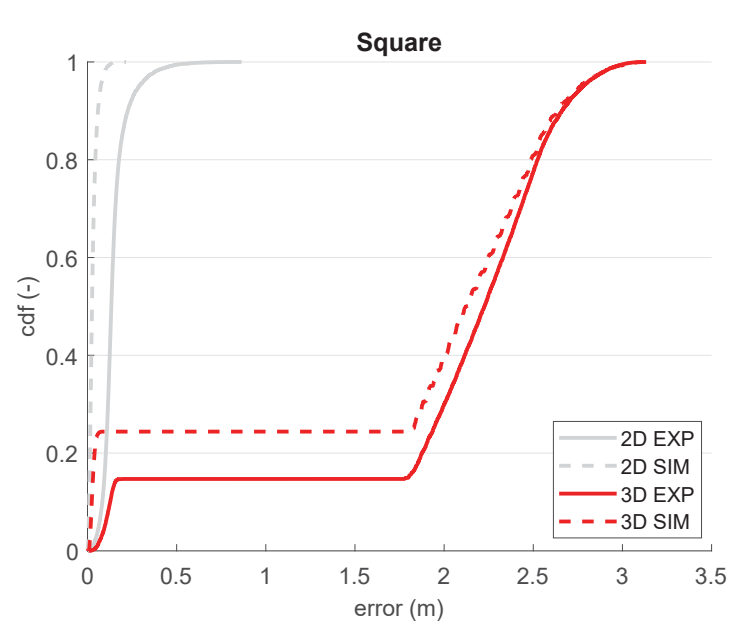

Fig. 5: Cdf of positioning error for square LED deployment, for $2 \mathrm{D} / 3 \mathrm{D}$ and obtained via simulations/experiment.

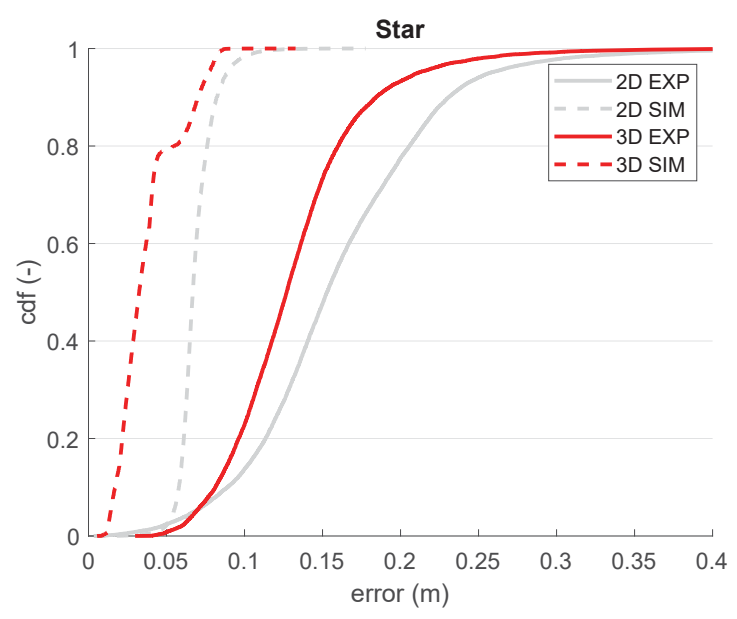

Fig. 6: Cdf of positioning error for star LED deployment, for 2D/3D and obtained via simulations/experiment.

mounted in a square shape, or formulated more generalized, when the fourth LED is located on the circle formed by the other three LEDs ('concyclic' LEDs). Fig. 5 shows that for this configuration, only about $20 \%$ of the locations are correctly matched, with accuracies comparable to the 2D estimations, while the other estimations are estimated with errors from around $1.75 \mathrm{~m}$ to more than $3 \mathrm{~m}$, due to a faulty height estimation. Similarly to the 2D case, the experimental results are slightly worse than the simulation results, but the cdfs exhibit a similar pattern and show errors in the same order of magnitude.

Fig. 7 shows the spatial distribution of the positioning errors for the square-shaped configuration according to a) simulations and $b$ ) experiments. The figure shows that correctly matched locations are randomly distributed in the evaluation area, and that the experiments have slightly fewer correct location matches.

Fig. 6 shows the simulated and experimental cdf of the 


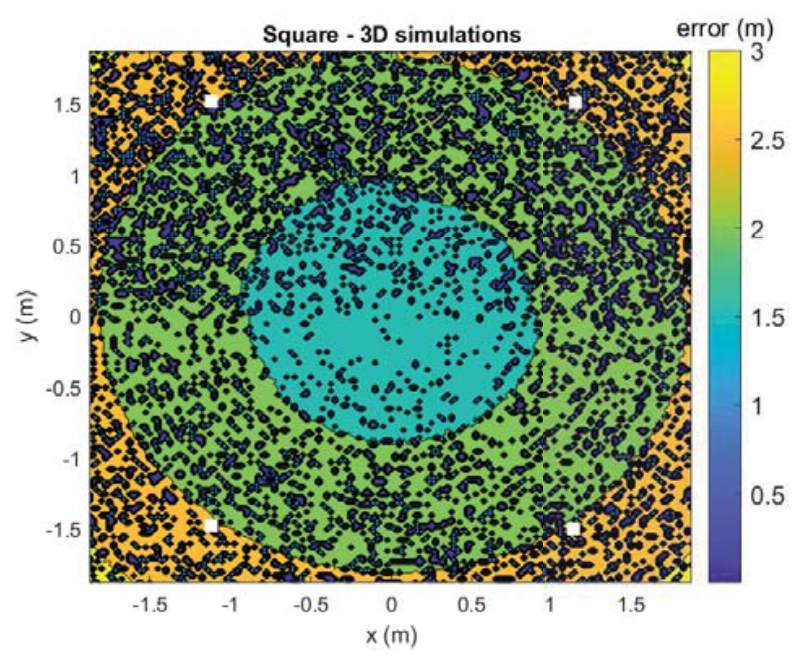

(a) Simulation

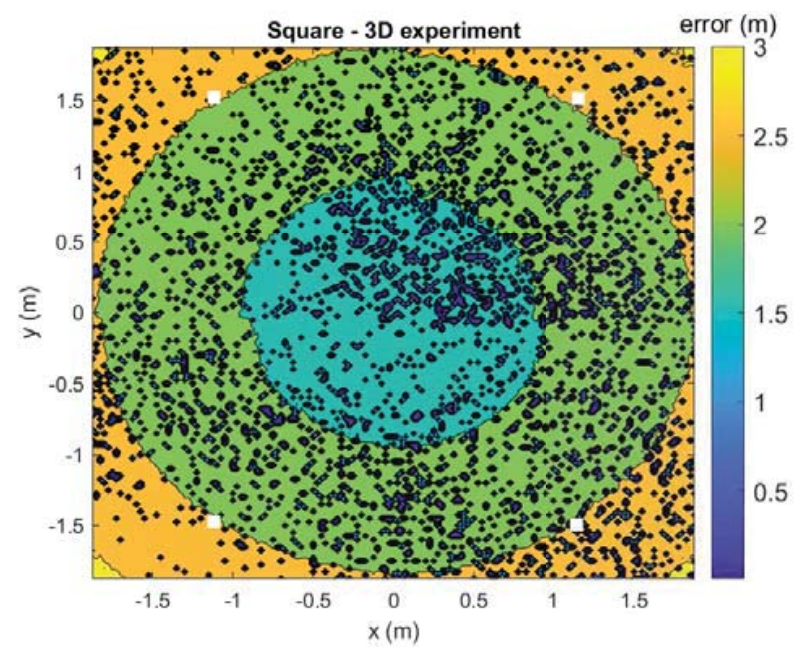

(b) Experiment

Fig. 7: Spatial distribution of positioning error for square-shaped LED configuration (white dots indicate LED locations)

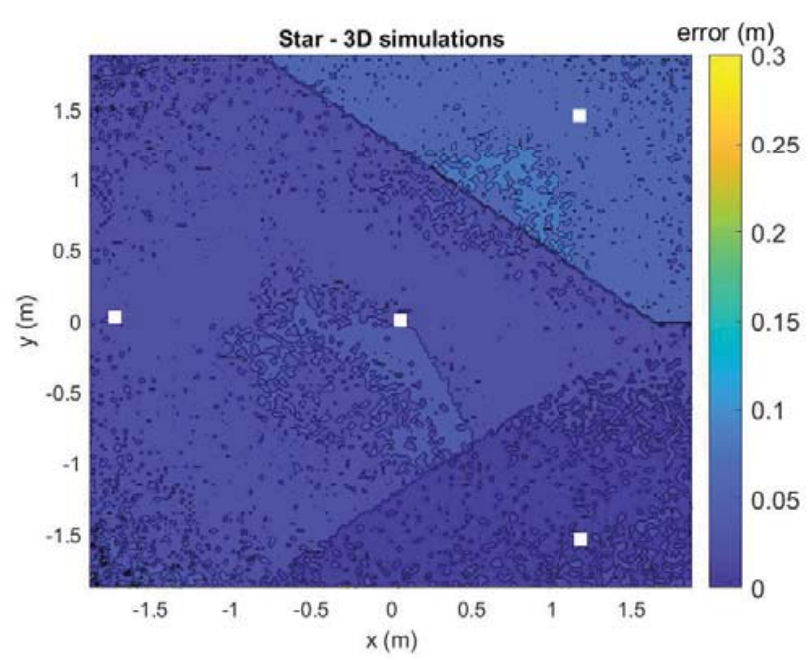

(a) Simulation

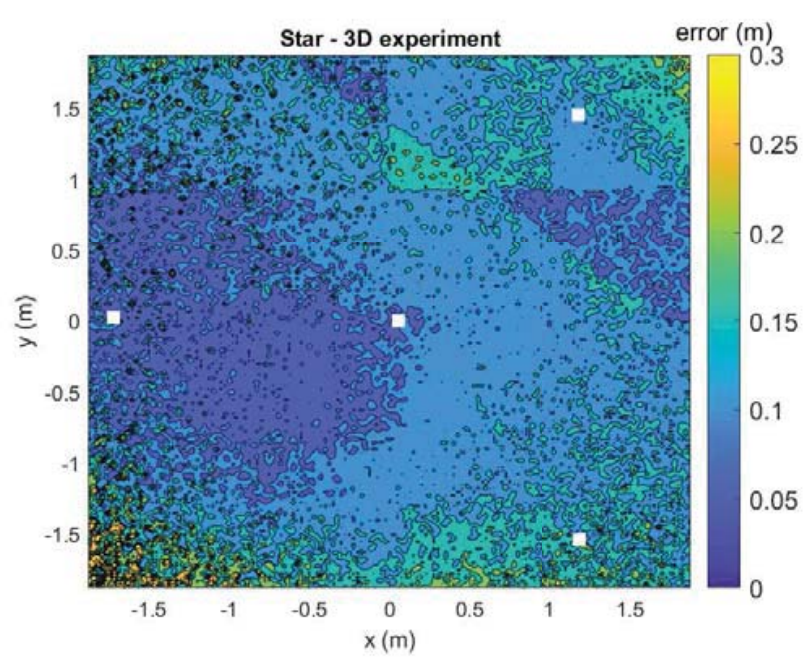

(b) Experiment

Fig. 8: Spatial distribution of positioning error for star-shaped LED configuration (white dots indicate LED locations)

positioning error using the star-shaped LED configuration in the same system, both for the 3D estimation and for the $2 \mathrm{D}$ estimation. The figure shows that the position ambiguity has indeed disappeared. Median and maximal experimental 3D errors are only $12.7 \mathrm{~cm}$ and $21.1 \mathrm{~cm}$ respectively, somewhat higher than the corresponding simulated errors $(3.2$ and $7.7 \mathrm{~cm}$ respectively). The $3 \mathrm{D}$ simulation cdf shows a small sudden increase at around $80 \%$, which is due to the smaller transmit power of the top right LED, and hence, the lower SNR. This can also be seen in Fig 8, showing the spatial distribution of the positioning errors for the star-shaped configuration according to a) simulations and b) experiments. Indeed, Fig 8 a) shows that positioning errors are slightly higher in the area around the top right LED with its slightly lower transmit power. It is also to be noted that $3 \mathrm{D}$ positioning performs better than 2D positioning (with a known PD height), although it uses the same $2 \mathrm{D}$ positioning algorithm. This can be explained by the fact that the $3 \mathrm{D}$ algorithm searches for a cost minimum in three dimensions, resulting in a better match than a search in only two dimensions. 
For both deployments ('square' and 'star'), it is observed that despite the correspondence of the nature of the error cdf of simulations and experiments, larger errors are observed in the experiments. These are due to several factors, e.g., the LEDs having a small unknown tilt [15], the LED power showing a deviation from the tabulated value [16], the LED radiation pattern not being perfectly Lambertian, the receiver angular responsivity not exhibiting a perfect cosine-dependence [17], small receiver tilts,... In the simulations, all these factors are assumed ideal, but in the experiments, they cannot be avoided.

\section{Vi. Conclusion}

In this paper, two different four-LED configurations were compared via simulations and experiments. It was shown that when the four LEDs are mounted in the corners of a square (i.e., concyclic locations), 3D positioning performance is inadequate due to a positioning ambiguity, with $80 \%$ of the locations matched with an error of more than $1.75 \mathrm{~m}$. For the star-shaped configuration, much better results were obtained: experimentally obtained median and maximal 3D positioning errors were 12.7 and $21.1 \mathrm{~cm}$, even better than the 15.3 and $25.7 \mathrm{~cm}$ when the exact height is accurately known (2D positioning). Future work consists of adjusting the algorithm to account for LED tilt or for receiver tilts, the latter of which is particularly important when applying the algorithm for drone navigation. Other future research consists of determining the optimal LED layout for a minimal threedimensional positioning error. Finally, it should further be investigated how the algorithm performs at different receiver heights. Realizing such 3D VLP system in industrial settings also requires investigating the impact of reflections and of external light sources.

\section{ACKNOWLEDGMENT}

The authors want to thank Matthias Van den Bossche for assisting during the realization of the measurement configuration.

\section{REFERENCES}

[1] J. Trogh, D. Plets, L. Martens, and W. Joseph, "Advanced real-time indoor tracking based on the viterbi algorithm and semantic data," International Journal of Distributed Sensor Networks, vol. 11, no. 10, 2015. [Online]. Available: http://dsn.sagepub.com/content/11/10/271818.abstract

[2] Y. Li, Z. Ghassemlooy, X. Tang, B. Lin, and Y. Zhang, "A vlc smartphone camera based indoor positioning system," IEEE Photonics Technology Letters, vol. 30, no. 13, pp. 1171-1174, July 2018.

[3] M. R. Mahfouz, C. Zhang, B. C. Merkl, M. J. Kuhn, and A. E. Fathy, "Investigation of high-accuracy indoor 3-d positioning using uwb technology," IEEE Transactions on Microwave Theory and Techniques, vol. 56, no. 6, pp. 1316-1330, June 2008 .

[4] N. Macoir, M. Ridolfi, J. Bauwens, B. Jooris, B. Van Herbruggen, J. Rossey, J. Hoebeke, and E. De Poorter, "Low power, portable and infrastructure light indoor uwb ranging solution: Demo," in Proceedings of the 18th International Conference on Information Processing in Sensor Networks, ser. IPSN '19. New York, NY, USA: ACM, 2019, pp. 337-339. [Online]. Available: http://doi.acm.org/10.1145/3302506.3312482

[5] S. Zhang, P. Du, C. Chen, W. Zhong, and A. Alphones, "Robust 3d indoor vlp system based on ann using hybrid rss/pdoa," IEEE Access, vol. 7, pp. 47 769-47 780, 2019.
[6] S. . Yang, E. . Jeong, D. . Kim, H. . Kim, Y. . Son, and S. . Han, "Indoor three-dimensional location estimation based on led visible light communication,” Electronics Letters, vol. 49, no. 1, pp. 54 -56, January 2013

[7] D. Plets, Y. Almadani, S. Bastiaens, M. Ijaz, L. Martens, and W. Joseph, "Efficient 3D trilateration algorithm for visible light positioning," Journal of Optics, 2019. [Online]. Available: http://iopscience.iop.org/10.1088/2040-8986/ab1389

[8] L. Zeng, D. C. O’Brien, H. L. Minh, G. E. Faulkner, K. Lee, D. Jung, Y. Oh, and E. T. Won, "High data rate multiple input multiple output (mimo) optical wireless communications using white led lighting," IEEE Journal on Selected Areas in Communications, vol. 27, no. 9, pp. 1654 1662, December 2009.

[9] T. Komine and M. Nakagawa, "Fundamental analysis for visible-light communication system using led lights," IEEE Transactions on Consumer Electronics, vol. 50, pp. 100-107, 2004.

[10] E. Amaldi, A. Capone, and F. Malucelli, "Planning umts base station location: optimization models with power control and algorithms," IEEE Transactions on Wireless Communications, vol. 2, no. 5, pp. 939-952, Sep. 2003.

[11] D. Plets, W. Joseph, K. Vanhecke, E. Tanghe, and L. Martens, "Coverage Prediction and Optimization Algorithms for Indoor Environments," EURASIP Journal on Wireless Communications and Networking, Special Issue on Radio Propagation, Channel Modeling, and Wireless, Channel Simulation Tools for Heterogeneous Networking Evaluation, vol. 1, 2012. [Online]. Available: http://jwcn.eurasipjournals.com/content/2012/1/123

[12] Z. Liu, W. Dai, and M. Z. Win, "Node placement for localization networks," in 2017 IEEE International Conference on Communications (ICC), May 2017, pp. 1-6.

[13] J. N. Ash and R. L. Moses, "On optimal anchor node placement in sensor localization by optimization of subspace principal angles," in 2008 IEEE International Conference on Acoustics, Speech and Signal Processing, March 2008, pp. 2289-2292.

[14] S. D. Lausnay, L. D. Strycker, J.-P. Goemaere, N. Stevens, and B. Nauwelaers, "A Visible Light Positioning system using Frequency Division Multiple Access with square waves," in 2015 9th International Conference on Signal Processing and Communication Systems (ICSPCS), Cairns, QLD, Australia, 14-16 Dec 2015, pp. 1-7.

[15] D. Plets, S. Bastiaens, L. Martens, and W. Joseph, "An analysis of the impact of led tilt on visible light positioning accuracy," Electronics, vol. 8, no. 4, 2019. [Online]. Available: http://www.mdpi.com/20799292/8/4/389

[16] D. Plets, S. Bastiaens, N. Stevens, L. Martens, and W. Joseph, "On the Impact of LED Power Uncertainty on the Accuracy of 2D and 3D Visible Light Positioning," Optik, 2019, accepted.

[17] S. Bastiaens, W. Raes, N. Stevens, W. Joseph, and D. Plets, "New photodiode responsivity model for rss-based vlp," in Global LiFi Congress Paris, France, June 2019, pp. 1-6. 